Running Head: RESEARCH DOMAIN CRITERIA

\title{
Operationalizing NIMH Research Domain Criteria (RDoC) in Naturalistic Clinical
}

\section{Settings}

Carla Sharp, ${ }^{\text {ab }}$ J. Christopher Fowler,${ }^{\text {c }}$ Ramiro Salas, ${ }^{\mathrm{c}}$ David Nielsen, ${ }^{\mathrm{cd}}$ Jon Allen, ${ }^{\mathrm{bc}}$ John Oldham, ${ }^{\text {bc }}$ Thomas Kosten, ${ }^{\text {cd }}$ Sanjay Matthew, ${ }^{\text {bc }}$ Alok Madan, ${ }^{\text {bc }}$ B. Christopher Frueh, ${ }^{\text {be }}$ Peter Fonagy

a University of Houston, Department of Psychology, Houston, TX, 77204

b The Menninger Clinic, 12301 Main Street, Houston, TX 77035

c Baylor College of Medicine, One Baylor Plaza, Houston, TX 77030

d Michael DeBakey Veterans Affairs Medical Center, 2002 Holcombe Blvd., Houston, TX 77030

e University of Hawaii, 200 Kawili St., Hilo, HI 96720

f University College of London, Gower St. London WC1E 6BT, UK

Correspondence: Carla Sharp, Ph.D. University of Houston, Department of Psychology, Houston, TX, 77204, csharp2@uh.edu

Acknowledgments: This work was partially supported by funding from The Menninger Clinic, The McNair Medical Institute, grants from NIH, and the Toomim Family Fund. We also acknowledge the contributions of Ian Aitken, Dr. Charles Neblett, and Dr. Stuart Yudofsky. This material is the result of work supported with resources and the use of facilities at the Michael E. DeBakey VA Medical Center, Houston, TX. 


\begin{abstract}
Recently, the National Institute of Mental Health (NIMH) introduced the Research Domain Criteria (RDoC) initiative to address two major challenges facing the field of psychiatry: (1) the lack of new effective personalized treatments for psychiatric disorders, and (2) the limitations associated with categorically-defined psychiatric disorders. While the potential of RDoC to revolutionize personalized psychiatric medicine and psychiatric nosology has been acknowledged, it is unclear how to implement $\mathrm{RDoC}$ in naturalistic clinical settings as part of routine outcomes research. In this paper we present the major RDoC principles and then show how these principles are operationalized in the Menninger Clinic's McNair Initiative for Neuroscience Discovery-Menninger \& Baylor College of Medicine (MIND-MB) study. We discuss how RDoC-informed outcomes-based assessment in clinical settings can transform personalized clinical care through multimodal treatments.
\end{abstract}




\section{The need for RDoC}

Effective treatments and policies are lacking for adolescents and adults with serious mental illness (SMI; i.e., treatment refractory neuropsychiatric disorders). Because tightly controlled efficacy studies restrict the capacity to provide personalized care, they may also restrict treatment response. Moreover, health insurance and public sector mental healthcare systems rarely allow for more than very brief psychiatric hospitalizations to focus primarily on stabilization. Thus, the majority of people with SMI are not afforded the time in treatment necessary to understand and treat their complex clinical symptoms and functional impairments. Offering personalized care means recognizing that for reasons of genetic makeup and personal history, people respond differentially to specific treatments and that poor response to one treatment does not necessarily imply poor response to another, or deny the possibility of potentiation through combinations of multimodal treatments over time.

In addition to the challenges facing personalized clinical care for SMI, the publication of the $5^{\text {th }}$ edition of the Diagnostic and Statistical Manual for Mental Disorders in 2013 by the American Psychiatric Association was preceded by controversy and contentious debate (Regier, 2007a, 2007b; Saunders, 2006; Widiger \& Simonsen, 2005). Despite the hopes that the practice of psychiatric diagnosis would be revolutionized, the knowledge base in the field was not judged sufficient to justify moving beyond the established tradition of diagnosing mental disorders based on clinical observation and patients' phenomenological symptom reports. In addition, the polythetic and dichotomous (categorical) diagnostic system was, for the most part, retained. In psychiatry (unlike other disciplines in medicine) we rely solely on the patient's subjective report, and on clinical observation, for diagnosis and treatment planning. In other words one could say that in psychiatry the disease is diagnosed as the symptom. Thus anxiety, for instance, is both the 
diagnosis and its symptom. In general medicine, by contrast, when a patient presents with excessive thirst and frequent restroom breaks, the physician might order a blood glucose level test to confirm or dispute a diagnosis of diabetes, acknowledging that the behavioral phenotype (excessive thirst and frequent restroom breaks) may be associated with a variety of underlying conditions and that a direct mapping of subjective patient report to pathophysiology may clarify the disorder. In psychiatry, when a patient presents with anger outbursts, affective instability, a history of suicide attempts, alcohol abuse and relationship problems, he/she meets five out of nine DSM-5 criteria for borderline personality disorder and the physician diagnoses the disorder without any information about underlying biological processes.

The DSM system furthermore conceptualizes diagnoses as polythetic dichotomies (Oldham, 2005). The polythetic nature of diagnoses means that patients can present with only a portion of the criteria that define a disorder and will receive a given diagnosis as long as the patient has met the symptom threshold (Silverman \& Krueger, 2014). Thus, staying with the example of borderline personality disorder, another patient may present with chronic feelings of emptiness, identity disturbance, paranoid ideation, abandonment fears and shoplifting and also meet criteria for borderline personality disorder. Indeed, there are 256 different combinations of symptoms that all result in a person receiving a diagnosis of borderline personality disorder (Skodol, Bender, Gunderson, \& Oldham, 2014). For PTSD in the DSM-IV there were 1,750 different combinations of symptoms that led to a diagnosis, and in DSM-5 it is over 10,000 (Rosen, Lilienfeld, Frueh, McHugh, \& Spitzer, 2010). Therefore, often, patients with the same diagnoses can have very different symptom patterns, thereby calling into question the validity of the disorder. Consequently, patients may be misdiagnosed or needlessly diagnosed with one or more comorbid disorders (Hyman, 2007). The patient described above presenting with anger 
outbursts, affective instability, a history of suicide attempts, alcohol abuse and relationship problems could potentially be diagnosed with bipolar II disorder or a substance use disorder or both. Conditions have such marked overlap in symptoms that similar features may count towards supposedly different diagnoses.

A dichotomous (categorical) diagnostic system utilizes a threshold of symptoms to establish a diagnosis. Thus, in our earlier example, a person must meet five (or more) out of nine criteria for borderline personality disorder. If a patient presented with a history of suicide attempts, affective instability and anger outbursts (three criteria) the patient would not receive a diagnosis. In this way, the diagnoses are considered as a yes/no distinction, with no room in the rubric for gradations of a disorder (Silverman \& Krueger, 2014), yet a real possibility exists that a patient would not receive needed services. Moreover, diagnostic thresholds utilized in the categorical approach often are arbitrary, while the variability both above and below diagnostic thresholds can be clinically meaningful (Kessler et al., 2003) and does not mirror the way practicing clinicians use the categories in everyday practice (Kim \& Ahn, 2002; Mussigbrodt et al., 2000; Reed, Mendonca Correia, Esparza, Saxena, \& Maj, 2011).

The current DSM system, characterized by polythetic and dichotomous (categorical), criteria-based diagnoses based on patients' phenomenological symptom reports, was implemented for the first time in the Third Edition of the Diagnostic and Statistical Manual (DSM-III) in 1980, and refined in DSM-IV-TR in 2000. In many ways, this diagnostic system has served clinical practice and research well (Silverman \& Krueger, 2014). We now have an improved understanding of many disorders and progress has been made in the development and dissemination of empirically validated and manualized treatments, allowing for improved quality of care for psychiatric patients. Accordingly, the diagnostic categories represented in the DSM-5 
still reflect contemporary consensus for the diagnosis and treatment of mental disorders, and are used in some countries for insurance billing, as standard requirements for medication trials, and in the U.S. as a predominant standard in the NIMH grant review process.

However, the reliance on polythetic and dichotomous (categorical) diagnoses at the level of the behavioral phenotype alone has impeded the use of advances in genomics, pathophysiology, psychopharmacology and behavioral science to aid in the diagnosis and treatment of psychiatric disorders (Insel et al., 2010; Insel, 2014). For example, the current funding crisis in the development of new psychoactive agents has been unequivocally attributed by some to inadequacies of the currently used psychiatric diagnostic system: "The data are in, and it is clear that a massive experiment has failed. A major barrier to progress is the current state of nosology in psychiatry. A new taxonomy is a prerequisite for meaningful progress. Today, few would argue that syndromes such as schizophrenia and depression are single, homogeneous diseases. And yet when it comes to clinical research, including clinical trials, both are still almost always treated as such" (Fibiger, 2012) (p.649). And while biological correlates of psychiatric disorders have been identified, variations in neurobiological systems or specific genes do not associate one-to-one with categorically defined mental disorders but, instead, cut across a variety of disorders. Moreover, it has also been argued that reliance on polythetic and dichotomous diagnoses may have led to a dehumanization of psychiatric practice by reducing the diagnostic process to a check of the presence or absence of symptoms (Maj, 2014).

One solution to this problem is to develop better diagnostic tests as we have argued elsewhere (Sharp, Monterosso, \& Montague, 2012). A complementary initiative would be to depart from the categorical classification system of mental disorders and to "develop new ways of classifying mental disorders based on dimensions of observable and neurobiological 
measures". Indeed, this statement is articulated in the NIMH strategic plan and led to the launching of the Research Domain Criteria Project (RDoC) in 2011 and has generated much discussion in the scientific literature (see for example Maj, 2014).

\section{The principles of RDoC}

RDoC is guided by three principles. First, in contrast to the categorical DSM system, $\mathrm{RDoC}$ is conceived as a dimensional system spanning the range from normal to abnormal. Second, RDoC is agnostic about DSM categories. RDoC seeks to "carve nature at its joints" by generating classifications stemming from basic behavioral neuroscience. Whereas psychiatry typically starts with a DSM diagnosis and then seeks neurobiological underpinnings, RDoC begins with current knowledge of behavior-brain relations and links them to clinical phenomena (Insel et al., 2010). RDoC uses multiple levels of analysis in studying functional domains from genes to the level of self-report. Finally, recruiting from large psychiatric clinics across a broad spectrum of psychiatric diagnoses to study, for example, fear circuitry provides an alternative to the recruitment of individuals with circumscribed symptom profiles (Insel et al., 2010).

To operationalize the above principles, RDoC provides a matrix with rows that cover five domains of function: Negative Valence Systems, Positive Valence Systems (approach/motivation), Cognitive Systems, Systems for Social Processes and Arousal/Regulatory Systems. Each of the domains is associated with relevant constructs selected for the potential that a particular brain circuit or area could reasonably be specified that implements that dimension of behavior. For instance, the Positive Valence Systems include the following constructs: reward valuation, effort valuation, reward expectancy/prediction error, initial responsiveness to reward, sustained responsiveness to reward, reward learning, and habit. The columns of the matrix 
represent seven different units of analysis including Genes, Molecules, Cells, Neural Circuits, Physiology, Behaviors and Self-report.

\section{Operationalizing $\mathrm{RDoC}$ in naturalistic clinical research settings}

While it is acknowledged that the problems with the current nosological system need to be addressed in naturalistic clinical settings (Maj, 2014), operationalizing RDoC principles in naturalistic clinical research settings can be challenging, partly because there is little precedent to draw from in psychiatry. NIMH has thus far approved about 40 RDoC studies. As the findings and methods of these studies are becoming public, we are developing a set of criteria that may guide the implementation of $\mathrm{RDoC}$ research in naturalistic clinical settings. Below, we outline these criteria, followed by a discussion of the implementation and progress of our project.

Dimensional approach to psychopathology. Studies examining the biological markers associated with psychiatric disorders have typically made use of case-control study designs using carefully screened and relatively small samples with little generalizability to actual psychiatric populations. In these studies it is unclear whether demonstrated group differences reflect symptom- or disorder-specific associations or nonspecific associations with latent dimensions of psychopathology. We must depart from research strategies that focus on a single diagnosis in isolation. Instead, the neurobiological basis of RDoC constructs must be examined using stateof-the-science models of dimensional structure of psychopathology in real-life psychiatric settings.

Recently, several models have been published of empirically defined dimensions of psychopathology that can be applied in this context. For instance, an internalizing-externalizing spectrum model has received considerable attention as a potential theoretical framework for understanding co-occurring psychiatric disorders (Krueger, 1999; Krueger, Caspi, Moffitt, \& 
Silva, 1998; Krueger, McGue, \& Iacono, 2001). Confirmatory factor analysis is used to fit measurement models for psychopathology that can be correlated with latent neural circuits or variables across different units of analyses. For this strategy, large sample sizes will be needed, as required by the latent trait models used to extract underlying psychopathology factors. It would also be important to make use of diagnostic tools that do not employ skip-out rules since the full criteria content will be used to extract factors.

Sampling. As discussed above, the over-reliance on case-control and randomized control trial study designs in psychiatry may partially explain the lack of progress in understanding underlying neurobiological processes associated with broad, cross-cutting dimensions of psychopathology. The field must develop a broader appreciation for scientific investigations utilizing representative psychiatric populations who present complex and multi-faceted symptom profiles, rather than relying so heavily on studies using carefully selected samples that bear little or no resemblance to real patients (Kessler et al., 2003). Therefore, actual patients in real-life settings need to be recruited for studies. In addition, because RDoC favors a dimensional system spanning the range from normal to abnormal, recruitment of real-life patient populations needs to be supplemented by the recruitment of non-treatment seeking individuals from the community. The aim is to capture the normal distribution of underlying biologically-based constructs rather than skewed distributions characteristic of only one end of the psychopathology spectrum.

Multiple units of analyses. Essential to any RDoC project is the collection of data across multiple units of analyses. For instance, DNA samples can be collected in order to test the specific impact of gene variants and epigenetics on psychopathology through the mediating effects of domain constructs. Conversely, studying a behavioral endophenotype such as rejection sensitivity should include performance-based assessment of behavior, neuroimaging, and 
genetics. A relatively novel area of research highlights the influence of gut microbiome on psychiatric disease (Foster \& Neufeld, 2013). Thus, a robust RDoC study may include the assessment of genes, systems that interact with the brain (e.g. gut microbes), circuits, behavior and self-report data.

Domains with the potential for cross-cutting associations across several psychiatric disorders. Essential to the success of an RDoC project would be choosing domains of function that have the potential for cross-cutting associations across several psychiatric disorders. For instance, the Positive Valence System is particularly interesting for thinking about divergence of the externalizing and internalizing factors underlying common mental disorders such that externalizing disorders are often associated with hyper-functioning of the Positive Valence System, while internalizing disorders are often associated with its hypofunctioning (Sharp et al., 2012). Because of these strong hypothesized links to psychopathology, and because the Positive Valence System is one of the research domain criteria most often associated with well-validated and well-used neuroimaging paradigms, this approach provides a tractable starting point for testing cross-cutting hypotheses.

\section{The potential for the availability of a public use data set for qualified research}

groups. Because an RDoC study would typically include a large sample of individuals unconstrained by traditional diagnostic boundaries, and data across multiple levels of analyses, an RDoC project should make available its data for analyses by other qualified research groups. In addition, the ideal RDoC study would provide a platform for future RDoC collaborations and extensions based on existing infrastructure.

Developmental aspects. Developmental influences on phenotypic plasticity need greater elucidation. Extant research on the structure of psychopathology focuses on individuals who 
report symptoms within a specified period. Ambiguity comes from mixing single-episode, oneoff cases with recurrent and chronic cases. These differ in the extent of their comorbid conditions, the severity of the conditions and potentially also the etiology of the conditions. This problem appears to be the case for depression, alcohol-use disorders and psychotic experiences (Jackson \& Sartor, in press; Monroe \& Harkness, 2011; van Os, Linscott, Myin-Germeys, Delespaul, \& Krabbendam, 2009). Comorbidity is sequential as well as cross-sectional. For instance, generalized anxiety disorder and major depressive disorder are linked to each other sequentially (each disorder increases the likelihood of developing the other) (Copeland, Shanahan, Costello, \& Angold, 2011; Kessler et al., 2011; Moffitt et al., 2007). In order to explain why some individuals who initially present with panic attacks go on to develop obsessive-compulsive disorder, bipolar disorder or psychosis we need to take account of both concurrent and sequential comorbidity when evaluating the structure of psychopathology. This requires a developmental approach.

Within a developmental approach, adolescence, which is defined as the transition phase between childhood and adulthood, is a developmental period of immense biological and social change (Nelson, Leibenluft, McClure, \& Pine, 2005). It is also a time of increased rates of accidents, alcohol and drug use, pregnancy, depression, suicide and violence (Crone \& Dahl, 2012). Adolescence is a time of increased risk for psychopathology as evidenced for instance by the National Comorbitidy Survey Replication $(N=9,000)$ that demonstrated that the peak age of onset for all mental disorders is 14 years (Kessler, Chiu, Demler, Merikangas, \& Walters, 2005). Evidence suggests that the increased risk for the emergence of psychopathology is related to anomalies or exaggerations of typical adolescent maturation processes in interaction with unique psychosocial or biological environments (Paus, Keshavan, \& Giedd, 2008). In addition, mental 
disorders are increasingly viewed as neurodevelopmental disorders (Dahl, 2004; Forbes \& Dahl, 2005), necessitating consideration of developmental aspects in the context of the RDoC framework. While prospective follow-up studies are ideal in this regard, important new information can be gleaned from studying RDoC constructs cross-sectionally across developmental epochs to identify unique neurobiological correlates of RDoC constructs.

Treatment outcome in naturalistic settings. RDoC holds great potential for identifying biomarkers and behavioral indicators for recovery from or improvement in mental illnesses. Biomarkers refer to characteristics that are measured objectively as an index of a pathogenic process or as a response to treatment (Carter et al.). While evidence is emerging in support of several domains of function (e.g. reward-related brain function as a predictor of treatment response in depression (Forbes et al.), these studies typically ignore the comorbidity of real-life psychiatric cases. Taking a dimensional cross-cutting approach to examining treatment response from the bottom-up (that is, brain function predicting treatment response across different disorders), would significantly move the field forward. For example, a recent study found that children with one or two copies of the nerve growth factor gene (NGF rs6330) T allele were significantly more likely to be free of their primary anxiety diagnosis after completing a course of cognitive behavioral therapy (Lester et al., 2012).

\section{Operationalizing RDoC in naturalistic treatment settings}

Consistent with the criteria above, in 2008 we developed a strategic vision for the research programs at The Menninger Clinic "to take advantage of the opportunity for longitudinal research, specifically to conduct important studies on clinical outcomes, disease course, economic aspects of mental illness, neuroimaging, genetics, and pharmacogenetics (p. 255)" (Frueh, Oldham, \& Fonagy, 2009), working with multidisciplinary research teams. In 
September 2014, this vision expanded to allow for the collection of fecal samples to better understand of the inter-relationship between gut biomarkers and psychiatric disease, illness burden, and/or response to treatment. Building on The Clinic's tradition of research, and with strong institutional commitment from leadership and collaborative relationships with investigators at Baylor College of Medicine (BCM) and the University of Houston, we developed a research program that fits into the $\mathrm{RDoC}$ rubrics and with its overarching goal, naming it the Menninger Clinic's McNair Initiative for Neuroscience Discovery-Menninger \& Baylor College of Medicine (MIND-MB) study. We are supported in this effort by an annual budget of approximately $\$ 2$ Million, with funds provided by The Menninger Clinic, the Department of Psychiatry at Baylor College of Medicine, the McNair Medical Institute, and many other local foundations and donors.

Research participants. The Menninger Clinic admits approximately 1,000 patients per year who typically stay for 6-8 weeks on one of 5 units. Over the course of 3 years, we aim to recruit and scan 1,500 inpatients at Menninger across adult and adolescent units to include 12-65 year olds (including approximately 210 adolescents), in addition to 250 patients from outpatient clinics in Houston, as well as 250 non-treatment seeking individuals from the community. There are no exclusion criteria for the genetic or gut microbiome portions of the study. Exclusion criteria for the fMRI protocol include the usual fMRI exclusions.

All patients admitted to The Menninger Clinic have the opportunity to participate in the study. Thus far, the response rate for participation to date is $73 \%$. Gender is equally distributed among adult inpatient units (50\% male, 50\% female), yet adult males volunteer and complete the study at a slightly higher rate (58\%) than females $(42 \%)$. The average age of adult subjects is $31.35(\mathrm{SD}=11.97)$. The adolescent sample is currently comprised of $46 \%$ males and $54 \%$ 
females with an average age of $15.54(\mathrm{SD}=1.61)$. Typical reasons for non-participation include disinterest, claustrophobia, and planned brief treatment.

fMRI task. Consistent with RDoC criteria as discussed above, we have elected a simple reward conditioning paradigm as the first task to examine domain function in a cross-cutting way. This task will elicit brain responses in several brain areas, but most importantly in the striatum. The striatum is the input structure of the basal ganglia and is responsible for the processing of affective stimuli such as rewards (Delgado, 2007). The striatum can be subdivided into two components. The dorsal striatum consists of the dorsal caudate nucleus and putamen, and receives extensive projections from dorsolateral prefrontal cortex as well as other surrounding frontal regions. The ventral striatum consists primarily of the nucleus accumbens, along with ventral portions of the caudate and putamen, and receives extensive projections from the ventral frontal regions. The ventral striatum also has extensive connections with limbic areas implicated in emotional processing. The dorsal and ventral striatum therefore are considered to be functionally distinct with the ventral striatum involved in affective and motivational processing while the dorsal striatum is involved in more cognitive or sensorimotor function (Delgado, 2007; Haber, Fudge, \& McFarland, 2000; Strathearn, 2011). Anomalies in activation of the striatum have been associated with both internalizing and externalizing disorders. The simple reward conditioning task selected have been shown to activate striatal activity (maximally in the putamen) (McClure, Berns, \& Montague, 2003) as well as the habenula (Salas, Baldwin, de Biasi, \& Montague, 2010), a brain center for the processing of negative events (Matsumoto \& Hikosaka, 2007). This task maps on to the RDoC domain constructs of positive (initial response to reward, sustained response to reward, expectancy/reward prediction error) and negative (frustrative non-reward) valence subdomains. 
To complement task-based fMRI we chose two techniques. First, we will perform Resting State Functional Connectivity (RSFC) to assess the functional connectivity among different areas of the brain (Biswal et al., 2010). RSFC anomalies associated with psychiatric disorders have been shown for depression (Guo et al., 2013) and addiction (Kelly et al., 2011). In a first round of analysis, we found a difference in inter-hemispherical insular RSFC in patients suffering from drug abuse, when compared to psychiatric patients that were matched for several parameters but did not suffer from drug abuse (Viswanath et al., 2015). Second, we will perform a Diffusion Tensor Imaging (DTI) experiment to measure white matter tracts in the brain. DTI will allow us to study the white matter tracts that connect different areas of the brain. RSFC and DTI data will be analyzed both as whole brain data driven comparisons and using regions of interest derived from the literature and from our own data (for example, results from the rewardrelated task may suggest regions of interest to study in RSFC and DTI).

Genetic data. Consistent with the RDoC principle of collecting data at multiple units of analysis, two blood draws are performed by either a trained assistant or a certified phlebotomist. All samples are transported to the Psychiatric Genetics Laboratory at BCM/Veterans Affairs Medical Center, except for the samples from adolescents. These samples are sent to the Rutgers Cell and DNA Repository in New Jersey where DNA will be extracted and lymphocytes cyropreserved. If we are unable to collect a blood sample, a saliva sample is used. The first draw occurs when a patient first enters the study and a second draw is taken at the conclusion of treatment. The second draw is done to serve as a quality control measure to confirm the identity of the first blood sample and to assess genotyping quality and reproducibility. In addition, the second sample will allow us in future studies to assess changes in epigenetic markers, such as 
DNA methylation and chromatin structure, which have been shown to change in response to the environment and may change in response to the therapy received at Menninger.

Initially, specific variants in several candidate genes that have been shown in human or animal studies to be involved in neurological pathways of psychiatric diseases, in vulnerability to develop a psychiatric disease, or in response to pharmacotherapy will be evaluated. In the first phase of the study, variants in the candidate genes will be genotyped and examined for association with the specified phenotypes and possible gene by environment interaction (GxE). A GxE study will be conducted to determine if the influence of the genetic variants on phenotype is different depending on patient history, such as childhood abuse. To avoid statistical bias due to population structure, data derived from ancestry informative markers will be evaluated for potential population. In the second phase of this study, gene effects and gene by environment interactions will be analyzed using next generation sequencing technology.

\section{Microbiome data}

Again, in line with $\mathrm{RDoC}$ principle of collecting data across multiple units of analysis, we collect fecal samples from adult and adolescent patients who agree to participate in this facet of the research project at Menninger. The microbes that reside in the human gastrointestinal tract form communities, and these communities differ among between people. Variations are determined by several factors such as nutrition or physiology and have an impact in health and disease, including psychiatric illness.

After collecting stool samples, nucleic acid (gDNA and/or RNA) will be isolated.

Nucleic acid samples will be sequenced on a variety of platforms (i.e. Roche 454, Illumina HiSeq) to generate sequence information, allowing for the identification of bacteria/viruses present in the samples. Gene expression studies will be confirmed by RT-PCR on RNA samples. Note, 
the nucleic acids will be stored until completion of the study and will be discarded in an appropriate manner at that time.

Diagnostic and symptom assessments. The Structured Clinical Interview for DSM-IV Disorders (SCID I; 44) and the Structured Clinical Interview for DSM-IV Personality Disorders (SCID-II; (First, Spitzer, Gibbon, Williams, \& Benjamin, 1994) are administered and all criteria individually coded without applying any skip-out rules. In addition, self-report and interviewbased assessments are completed at admission with symptom and functional measures repeated at 2 week intervals during hospitalization and at post-discharge follow-up. All psychiatric assessment measures are integrated into treatment planning and monitoring of patient care as an element of quality improvement. Constructs assessed include: functional disability and wellbeing (Bech, Olsen, Kjoller, \& Rasmussen, 2003; Ustun, Kostanjek, Chatteerji, \& Rehm, 2010), substance abuse (Organization, 2010), suicide-related behaviors (Posner et al., 2011), depression, anxiety and somatization (Kroenke, Spitzer, Williams, \& Lowe, 2010), affect regulation (Gratz \& Roemer, 2004), and personality traits of the five factor model (John, Naumann, \& Soto, 2008).

Self-report assessments of Positive Valence System. Several self-report measures of Positive Valence Systems are included in the battery. The Barratt Impulsiveness Scale (Barratt, 1959), 11th revision (Patton, Stanford, \& Barratt, 1995) is a 30-item self-report instrument designed to assess the personality/behavioral construct of impulsiveness. The Behavioral Inhibition Scales/Behavioral Activation Scales (Carver \& White, 1994) is a self-report measure consisting of 20 questions that traditionally comprise 4 separate scales: behavioral inhibition, behavioral activation (goal-directed motivation), reward responsivenss (response upon receipt of reward), and fun-seeking (desire for novel rewards). 
Procedures. Figure 1 summarizes the recruitment, assessment and scanning of patients and community participants.

\section{Figure 1}

This broad-based multidimensional assessment approach of overarching domains of psychological functioning is particularly relevant to the RDoC. In addition the study was designed to assess the potential neurocognitive and epigenetic effects of an intensive course of treatment. A second fMRI of RSN, DTI and task-related functional imaging allows for assessment of change and resting state networks as well as the major neural circuits including those of disappointment and reward, positive and negative valence systems, and social cognition. A second blood draw at 21 days after the initial blood draw (pending IRB approval) will allow us to assess potential epigenetic changes by studying the methylation of targeted genes.

\section{Concluding remarks}

The validity of DSM-based psychiatric disorders has been called into question (Kendell, 1989) providing the rationale for RDoC. This is not to say that classification of all the major psychiatric disorders is arbitrary. Cross-cultural similarities in symptom expression and evidence for heritability suggest that DSM criteria identify real conditions, even if they do so imprecisely (Hyman, 2007). And it is also not to say that RDoC is not without its limitations. For example, RDoC constructs, as defined, appear to be quite distant from clinical phenomena, potentially rendering them of limited value in describing or defining psychopathology (Maj, 2014). Related, the relationship between $\mathrm{RDoC}$ philosophy and clinical reality is unclear; for example, patients do not present to the psychiatrist with their genotype or biosignature, but with their phenotype (Jablensky \& Waters, 2014). Thus, until biology has cultural currency, we will to some extent always be relying on the subjective accounts of our patients' symptoms as a first line of inquiry. 
However, there is room for improvement, but these changes need to be empirically motivated. In this paper, we have provided an example of implementing RDoC aims in a naturalistic clinical research setting. Successful implementation of this program of research could provide important public access data on the correlates of mental disorder and treatment response across and within domains of functioning.

Our objective is to develop clinically valid dimensions of individual variation, and clusters of individuals (population sub-groups with common trajectories of ontogenesis) that provide a much greater fit with underlying neural networks and neurocognitions than has been achieved. We will pursue a data-driven (bottom-up) multidimensional approach to improve the precision of clinical measurement. We recognize that a multidimensional approach, however descriptive, will remain essentially unhelpful if it cannot be applied to prediction of such things as course of illness and treatment outcome. Accumulating evidence suggests that disorders are positively correlated not just at the disorder level (comorbidity/co-occurrence), but substantially so at the spectrum level (Externalizing and Internalizing spectra are correlated at around $\mathrm{r}=0.5$ and the Internalizing and Thought Disorder spectra at $r=0.6$ ). Thus, it seems that propensities to specific forms of psychopathology (e.g., Internalizing vs. Externalizing) and propensity to develop any and all forms of common psychopathologies may be inherited or acquired and biological studies informed by RDoC must aim to identify such generic propensities as well as spectra and syndrome level mechanisms (Caspi et al., 2014; Jablensky \& Waters, 2014). As some of these underlying correlations may be more apparent when individuals are studied over time, we consider it essential to adopt a developmental approach and consider systematic sampling across age groups as a critical feature of our research protocol. Characterizing specific domains within clinical phenotypes over time of treatment and follow-up enhances precision and 
validity. This sentiment is consistent with recent reflection on the clinical utility of the RDoC framework which suggests that RDoC will achieve its ultimate goal only if can produce a set of behavioral and biological measures whose reliability and sensitivity and specificity in predicting treatment (and other outcomes) improves over current symptom-based measures (Cuthbert, 2014; Maj, 2014).

It is important to note that a neural mechanisms-oriented approach such as we suggest here is perfectly consistent with the person-centered approach which is increasingly replacing the 'horse race' paradigm embodied in traditional RCT methodology. A person-centered neural mechanism-based approach in line with the emerging personalized medicine movement within psychiatry (Myers \& Nemeroff, 2010; Ozomaro, Wahlestedt, \& Nemeroff, 2013), may be able to answer the 'What works for whom, how, and under what circumstances?' question which diagnosis based approaches have sadly failed to fully elucidate (Fonagy et al., in press; Roth \& Fonagy, 2005).

We hope that the approach recommended here will help overcome the lack of progress not only in psychopharmacology, but the rather similar picture which is emerging from the psychosocial treatment literature. Since 1975 , meta-analyses have not found it easy to show superiority for any bona fide psychotherapy over any other and the literature has not highlighted specific techniques that account for change, and so called common factors account for a considerable proportion of the observed variance in outcome (American Psychological Association, 2012; Benish, Imel, \& Wampold, 2008; Imel, Wampold, Miller, \& Fleming, 2008; Lutz, Leon, Martiniwitch, Lyons, \& Stiles, 2007; Miller, Wampold, \& Varhely, 2008; Spielmans, Pasek, \& McFall, 2007; Zuroff, Kelly, Leybman, Blatt, \& Wampold, 2010). Underlying this lack of specificity lies a paradox, namely that for the findings of RCTs to be 
generalizable they have to gather samples that resemble the 'normal' clinical population (effectiveness studies), but for the findings to be replicable and interpretable the sample should not be obscured by comorbidity and concurrently administered treatments (efficacy studies). The heterogeneity of even such 'pure' samples in terms of the likely underlying causes leads treatment developers to take somewhat of a scatter gun approach trying to address a wide range of problems as part of a single manualized treatment package, much of which may not be relevant to the problems presented by a specific patient.

Taken together, $\mathrm{RDoC}$ has the potential to revolutionize not only our diagnostic nosology, but our ability to more effectively treat our patients. While no system is perfect, RDoC may provide a useful complement to the current diagnostic system (First, 2014; Maj, 2014) and our hope is that a research program as described here would facilitate a type of cross-walk between traditional and alternative approaches to psychopathology. Mostly, it is our hope that MIND-MB will uncover the multiple multi-modal pathways to recovery, thereby informing personalized psychiatric medicine for patients and families. 


\section{References}

American Psychological Association. (2012). Recognition of Psychotherapy Effectiveness. Retrieved 2nd May 2013, 2013, from http://www.apa.org/about/policy/resolutionpsychotherapy.aspx

Barratt, E. S. (1959). Anxiety and impulsiveness related to psychomotor efficiency. Perceptual and Motor Skills, 9, 191 - 198.

Bech, P., Olsen, L. R., Kjoller, M., \& Rasmussen, N. K. (2003). Measuring well-being rather than the absence of distress symptoms: A comparison of the SF-36 mental health subscale and the WHO-Five well-being scale. International Journal of Methods in Psychiatric Research, 12(2), 85-91. doi: Doi 10.1002/Mpr.145

Benish, S. G., Imel, Z. E., \& Wampold, B. E. (2008). The relative efficacy of bona fide psychotherapies for treating post-traumatic stress disorder: a meta-analysis of direct comparisons. Clin Psychol Rev, 28(5), 746-758.

Biswal, B. B., Mennes, M., Zuo, X. N., Gohel, S., Kelly, C., Smith, S. M., . . Milham, M. P. (2010). Toward discovery science of human brain function. [Multicenter Study Research Support, N.I.H., Extramural Research Support, Non-U.S. Gov't]. Proc Natl Acad Sci U S A, 107(10), 4734-4739. doi: 10.1073/pnas.0911855107

Carter, C. S., Barch, D. M., Bullmore, E., Breiling, J., Buchanan, R. W., Butler, P., ... Wykes, T. Cognitive Neuroscience Treatment Research to Improve Cognition in Schizophrenia II: Developing Imaging Biomarkers to Enhance Treatment Development for Schizophrenia and Related Disorders. Biol Psychiatry. doi: S0006-3223(11)00207-1 [pii] 10.1016/j.biopsych.2011.01.041 
Carver, C. S., \& White, T. L. (1994). Behavioral-Inhibition, Behavioral Activation, and Affective Responses to Impending Reward and Punishment - the Bis Bas Scales. Journal of Personality and Social Psychology, 67(2), 319-333. doi: Doi 10.1037//00223514.67.2.319

Caspi, A., Houts, R. M., WBelsky, D. W., Goldman-Mellor, S. J., Harrington, H., Israel, S., . . Moffit, T. E. (2014). The p factor: One general psychopathology factor in the structure of psychiatric disorders? Clinical Psychological Science, 2(2), 119-137.

Copeland, W., Shanahan, L., Costello, E. J., \& Angold, A. (2011). Cumulative Prevalence of Psychiatric Disorders by Young Adulthood: A Prospective Cohort Analysis From the Great Smoky Mountains Study. Journal of the American Academy of Child and Adolescent Psychiatry, 50(3), 252-261. doi: DOI 10.1016/j.jaac.2010.12.014

Crone, E. A., \& Dahl, R. E. (2012). Understanding adolescence as a period of social-affective engagement and goal flexibility. Nature Reviews Neuroscience, 13(9), 636-650. doi: Doi $10.1038 / \mathrm{Nrn} 3313$

Cuthbert, B. N. (2014). The RDoC framework: facilitating transition from ICD/DSM to dimensional approaches that integrate neuroscience and psychopathology. World Psychiatry, 13(1), 28-35. doi: 10.1002/wps.20087

Dahl, R. E. (2004). Adolescent brain development: A period of vulnerabilities and opportunities - Keynote address. Adolescent Brain Development: Vulnerabilities and Opportunities, 1021, 1-22. doi: DOI 10.1196/annals.1308.001

Delgado, M. R. (2007). Reward-related responses in the human striatum. Reward and Decision Making in Corticobasal Ganglia Networks, 1104, 70-88. doi: DOI 10.1196/annals. 1390.002 
Fibiger, H. C. (2012). Psychiatry, The Pharmaceutical Industry, and The Road to Better Therapeutics. Schizophrenia Bulletin, 38(4), 649-650. doi: DOI 10.1093/schbul/sbs073

First, M., Spitzer, R., Gibbon, M., Williams, J., \& Benjamin, L. (1994). Structured Clinical Interview for DSM-IV Axis II personality disorders (SCID II). New York: Biometric Research Department.

First, M. B. (2014). Preserving the clinician-researcher interface in the age of RDoC: the continuing need for DSM-5/ICD-11 characterization of study populations. World Psychiatry, 13(1), 53-54. doi: 10.1002/wps.20107

Fonagy, P., Cottrell, D., Philips, J., Bevington, D., Glaser, D. E., \& Allison, E. (in press). What Works For Whom? A Critical Review of Treatments for Children and Adolescents (2nd ed.). New York: Guilford.

Forbes, E. E., \& Dahl, R. E. (2005). Neural systems of positive affect: relevance to understanding child and adolescent depression? Dev Psychopathol, 17(3), 827-850. doi: S095457940505039X [pii] 10.1017/S095457940505039X

Forbes, E. E., Olino, T. M., Ryan, N. D., Birmaher, B., Axelson, D., Moyles, D. L., \& Dahl, R. E. Reward-related brain function as a predictor of treatment response in adolescents with major depressive disorder. Cogn Affect Behav Neurosci, 10(1), 107-118. doi: 10/1/107 [pii] 10.3758/CABN.10.1.107

Foster, J. A., \& Neufeld, K. A. M. (2013). Gut-brain: how the microbiome influences anxiety and depression. Trends in Neurosciences, 36(5), 305-312. doi: DOI 10.1016/j.tins.2013.01.005 
Frueh, B. C., Oldham, J. M., \& Fonagy, P. (2009). Programmatic research at a specialty psychiatric inpatient clinic: Opportunities, challenges, and future directions. Bulletin of the Menninger Clinic, 73(4), 251-258.

Gratz, K. L., \& Roemer, L. (2004). Multidimensional assessment of emotion regulation and dysregulation: Development, factor structure and initial validation of the Difficulties in Emotion Regulation Scale. Journal of Psychopathology and Behavioral Assessment, 26(1), 41-54.

Guo, W. B., Liu, F., Xue, Z. M., Gao, K. M., Liu, Z. N., Xiao, C. Q., . . Zhao, J. P. (2013). Decreased Interhemispheric Coordination in Treatment-Resistant Depression: A RestingState fMRI Study. Plos One, 8(8). doi: ARTN e71368 DOI 10.1371/journal.pone.0071368

Haber, S. N., Fudge, J. L., \& McFarland, N. R. (2000). Striatonigrostriatal pathways in primates form an ascending spiral from the shell to the dorsolateral striatum. [Research Support, U.S. Gov't, P.H.S.]. Journal of Neuroscience, 20(6), 2369-2382.

Hyman, S. E. (2007). Opinion - Can neuroscience be integrated into the DSM-V? Nature Reviews Neuroscience, 8(9), 725-U716. doi: Doi 10.1038/Nrn2218

Imel, Z. E., Wampold, B. E., Miller, S. D., \& Fleming, R. R. (2008). Distinctions without a difference: direct comparisons of psychotherapies for alcohol use disorders. Psychol Addict Behav, 22(4), 533-543.

Insel, T., Cuthbert, B., Garvey, M., Heinssen, R., Pine, D. S., Quinn, K., . . Wang, P. (2010). Research domain criteria (RDoC): toward a new classification framework for research on mental disorders. Am J Psychiatry, 167(7), 748-751. doi:

10.1176/appi.ajp.2010.09091379 
Insel, T. R. (2014). The NIMH Research Domain Criteria (RDoC) Project: precision medicine for psychiatry. Am J Psychiatry, 171(4), 395-397. doi: 10.1176/appi.ajp.2014.14020138

Jablensky, A., \& Waters, F. (2014). RDoC: a roadmap to pathogenesis? World Psychiatry, 13(1), 43-44. doi: 10.1002/wps.20100

Jackson, K. M., \& Sartor, C. E. (in press). The natural course of substance use and dependence. In K. J. Sher (Ed.), Oxford handbook of substance use disorders. . New York, NY: Oxford University Press.

John, O. P., Naumann, L. P., \& Soto, C. J. (2008). Paradigm Shift to the Integrative Big-Five Trait Taxonomy, History, Measurement, and Conceptual Issues. In O. P. John \& R. W. P. Robins, L. A. (Eds.), Handbook of personality: Theory and Research (pp. 114-158). New York, NY: Guilford Press.

Kelly, C., Zuo, X. N., Gotimer, K., Cox, C. L., Lynch, L., Brock, D., . . Milham, M. P. (2011). Reduced Interhemispheric Resting State Functional Connectivity in Cocaine Addiction. Biological Psychiatry, 69(7), 684-692. doi: DOI 10.1016/j.biopsych.2010.11.022

Kendell, R. E. (1989). Clinical validity. [Review]. Psychol Med, 19(1), 45-55.

Kessler, R. C., Chiu, W. T., Demler, O., Merikangas, K. R., \& Walters, E. E. (2005). Prevalence, severity, and comorbidity of 12-month DSM-IV disorders in the National Comorbidity Survey Replication. Arch Gen Psychiatry, 62(6), 617-627. doi: 62/6/617 [pii] 10.1001/archpsyc.62.6.617

Kessler, R. C., Merikangas, K. R., Berglund, P., Eaton, W. W., Koretz, D. S., \& Walters, E. E. (2003). Mild disorders should not be eliminated from the DSM-V. [Research Support, Non-U.S. Gov't 
Research Support, U.S. Gov't, P.H.S.]. Arch Gen Psychiatry, 60(11), 1117-1122. doi: 10.1001/archpsyc.60.11.1117

Kessler, R. C., Ormel, J., Petukhova, M., McLaughlin, K. A., Green, J. G., Russo, L. J., . . Ustun, T. B. (2011). Development of lifetime comorbidity in the World Health Organization world mental health surveys. [Research Support, N.I.H., Extramural Research Support, Non-U.S. Gov't

Research Support, U.S. Gov't, P.H.S.]. Arch Gen Psychiatry, 68(1), 90-100. doi: 10.1001/archgenpsychiatry.2010.180

Kim, N. S., \& Ahn, W. K. (2002). Clinical psychologists' theory-based representations of mental disorders predict their diagnostic reasoning and memory. [Comparative Study Research Support, Non-U.S. Gov't Research Support, U.S. Gov't, Non-P.H.S. Research Support, U.S. Gov't, P.H.S.]. J Exp Psychol Gen, 131(4), 451-476.

Kroenke, K., Spitzer, R. L., Williams, J. B., \& Lowe, B. (2010). The Patient Health Questionnaire Somatic, Anxiety, and Depressive Symptom Scales: a systematic review. [Research Support, Non-U.S. Gov't Review]. Gen Hosp Psychiatry, 32(4), 345-359. doi: 10.1016/j.genhosppsych.2010.03.006 Krueger, R. F. (1999). The structure of common mental disorders. [Research Support, Non-U.S. Gov't Research Support, U.S. Gov't, P.H.S.]. Arch Gen Psychiatry, 56(10), 921-926.

Krueger, R. F., Caspi, A., Moffitt, T. E., \& Silva, P. A. (1998). The structure and stability of common mental disorders (DSM-III-R): a longitudinal-epidemiological study. [Research Support, Non-U.S. Gov't Research Support, U.S. Gov't, Non-P.H.S. Research Support, U.S. Gov't, P.H.S.]. J Abnorm Psychol, 107(2), 216-227. 
Krueger, R. F., McGue, M., \& Iacono, W. G. (2001). The higher-order structure of common DSM mental disorders: internalization, externalization, and their connections to personality. Personality and Individual Differences, 30(7), 1245-1259. doi: Doi $10.1016 / \mathrm{S} 0191-8869(00) 00106-9$

Lester, K. J., Hudson, J. L., Tropeano, M., Creswell, C., Collier, D. A., Farmer, A., . . Eley, T. C. (2012). Neurotrophic gene polymorphisms and response to psychological therapy. Translational Psychiatry, 2. doi: ARTN e108 DOI 10.1038/tp.2012.33

Lutz, W., Leon, S. C., Martiniwitch, Z., Lyons, J. S., \& Stiles, W. B. (2007). Therapist effects in outpatient psychotherapy: A three-level growth curve aproach. Journal of Counselling Psychology, 54, 32-39.

Maj, M. (2014). Keeping an open attitude towards the RDoC project. [Editorial]. World Psychiatry, 13(1), 1-3. doi: 10.1002/wps.20111

Matsumoto, M., \& Hikosaka, O. (2007). Lateral habenula as a source of negative reward signals in dopamine neurons. Nature, 447(7148), 1111-1115. doi: 10.1038/nature05860

McClure, S. M., Berns, G. S., \& Montague, P. R. (2003). Temporal prediction errors in a passive learning task activate human striatum. Neuron, 38(2), 339-346. doi: Doi 10.1016/S08966273(03)00154-5

Miller, S., Wampold, B., \& Varhely, K. (2008). Direct comparisons of treatment modalities for youth disorders: a meta-analysis. Psychother Res, 18(1), 5-14.

Moffitt, T. E., Harrington, H., Caspi, A., Kim-Cohen, J., Goldberg, D., Gregory, A. M., \& Poulton, R. (2007). Depression and generalized anxiety disorder: cumulative and sequential comorbidity in a birth cohort followed prospectively to age 32 years. 
[Comparative Study Research Support, N.I.H., Extramural Research Support, Non-U.S. Gov't]. Arch Gen Psychiatry, 64(6), 651-660. doi: 10.1001/archpsyc.64.6.651

Monroe, S. M., \& Harkness, K. L. (2011). Recurrence in major depression: a conceptual analysis. Psychol Rev, 118(4), 655-674. doi: 10.1037/a0025190

Mussigbrodt, H., Michels, R., Malchow, C. P., Dilling, H., Munk-Jorgensen, P., \& Bertelsen, A. (2000). Use of the ICD-10 classification in psychiatry: an international survey. Psychopathology, 33(2), 94-99. doi: 29127

Myers, A. J., \& Nemeroff, C. B. (2010). New Vistas in the Management of Treatment-Refractory Psychiatric Disorders: Genomics and Personalized Medicine. FOCUS: The Journal of Lifelong Learning in Psychiatry, 8(4), 525-535.

Nelson, E. E., Leibenluft, E., McClure, E. B., \& Pine, D. S. (2005). The social re-orientation of adolescence: A neuroscience perspective on the process and its relation to psychopathology. Psychological Medicine, 35(2), 163-174.

Oldham, J. M. (2005). Personality disorders. Focus, 3, 372-382.

World Health Organization (2010). WHO-ASSIST The alcohol, smoking and substance involvement screening test (ASSIST): Manual for use in primary care Retrieved November 6, 2012, from http://whqlibdoc.who.int/publications/2010/9789241599382_eng.pdf

Ozomaro, U., Wahlestedt, C., \& Nemeroff, C. B. (2013). Personalized medicine in psychiatry: problems and promises. BMC Med, 11, 132. doi: 10.1186/1741-7015-11-132

Patton, J. H., Stanford, M. S., \& Barratt, E. S. (1995). Factor structure of the Barratt impulsiveness scale. Journal of Clinical Psychology, 51(6), 768-774. 
Paus, T., Keshavan, M., \& Giedd, J. N. (2008). Why do many psychiatric disorders emerge during adolescence? [Research Support, N.I.H., Extramural Research Support, Non-U.S. Gov't Review]. Nature Reviews Neuroscience, 9(12), 947-957. doi: 10.1038/nrn2513

Posner, K., Brown, G. K., Stanley, B., Brent, D. A., Yershova, K. V., Oquendo, M. A., . . Mann, J. J. (2011). The Columbia-Suicide Severity Rating Scale: initial validity and internal consistency findings from three multisite studies with adolescents and adults. [Multicenter Study Research Support, N.I.H., Extramural Research Support, Non-U.S. Gov't]. Am J Psychiatry, 168(12), 1266-1277. doi: 10.1176/appi.ajp.2011.10111704

Reed, G. M., Mendonca Correia, J., Esparza, P., Saxena, S., \& Maj, M. (2011). The WPA-WHO Global Survey of Psychiatrists' Attitudes Towards Mental Disorders Classification. World Psychiatry, 10(2), 118-131.

Regier, D. A. (2007a). Dimensional approaches to psychiatric classification: refining the research agenda for DSM-V: an introduction. International Journal of Methods in Psychiatric Research, 16, S1-S5. doi: Doi 10.1002/Mpr.209

Regier, D. A. (2007b). Time for a fresh start? Rethinking psychosis in DSM-V. Schizophrenia Bulletin, 33(4), 843-845. doi: DOI 10.1093/schbul/sbm055

Rosen, G. M., Lilienfeld, S. O., Frueh, B. C., McHugh, P. R., \& Spitzer, R. L. (2010). Reflections on PTSD's future in DSM-V. British Journal of Psychiatry, 197(5), 343-344. doi: DOI 10.1192/bjp.bp.110.079699

Roth, A., \& Fonagy, P. (2005). What works for whom? A critical review of psychotherapy research (2nd ed.). New York: Guilford Press. 
Salas, R., Baldwin, P., de Biasi, M., \& Montague, P. R. (2010). BOLD Responses to Negative Reward Prediction Errors in Human Habenula. Front Hum Neurosci, 4, 36. doi: 10.3389/fnhum.2010.00036

Saunders, J. B. (2006). Substance dependence and non-dependence in the Diagnostic and Statistical Manual of Mental Disorders (DSM) and the International Classification of Diseases (ICD): can an identical conceptualization be achieved? Addiction, 101, 48-58. doi: DOI 10.1111/j.1360-0443.2006.01589.x

Sharp, C., Monterosso, J., \& Montague, R. (2012). Neuroeconomics: A bridge for translational research. Biological Psychiatry.

Silverman, M. H., \& Krueger, R. F. (2014). Borderline personality disorder and DSM-5: New directions and hopes for the future. In C. Sharp \& J. Tackett (Eds.), Handbook of Borderline Personality Disorder in Children and Adolescents (pp. 433-450). New York, NY: Springer.

Skodol, A. E., Bender, D. S., Gunderson, J. G., \& Oldham, J. M. (2014). Personality Disorders. In R. Hales, S. C. Yudofsky \& L. Roberts (Eds.), American Psychiatric Press Textbook of Psychiatry (6th ed.). Arlington, VA: American Psychiatric Press.

Spielmans, G. I., Pasek, L. F., \& McFall, J. P. (2007). What are the active ingredients in cognitive and behavioral psychotherapy for anxious and depressed children? A metaanalytic review. Clin Psychol Rev, 27(5), 642-654.

Strathearn, L. (2011). Maternal neglect: oxytocin, dopamine and the neurobiology of attachment. J Neuroendocrinol, 23(11), 1054-1065. doi: 10.1111/j.1365-2826.2011.02228.x 
Ustun, T. B., Kostanjek, N., Chatteerji, S., \& Rehm, J. (2010). Measuring health and disability: Manual for the WHO Disability Assessment Schedule WHODAS 2.0. Geneva, Switzerland: World Health Organization Press.

van Os, J., Linscott, R. J., Myin-Germeys, I., Delespaul, P., \& Krabbendam, L. (2009). A systematic review and meta-analysis of the psychosis continuum: evidence for a psychosis proneness-persistence-impairment model of psychotic disorder. Psychol Med, 39(2), 179-195. doi: 10.1017/S0033291708003814

Widiger, T. A., \& Simonsen, E. (2005). Introduction to the special section: The American psychiatric Association's research agenda for the DSM-V. Journal of Personality Disorders, 19(2), 103-109. doi: DOI 10.1521/pedi.19.2.103.62626

Zuroff, D. C., Kelly, A. C., Leybman, M. J., Blatt, S. J., \& Wampold, B. E. (2010). Betweentherapist and within-therapist differences in the quality of the therapeutic relationship: effects on maladjustment and self-critical perfectionism. Journal of clinical psychology, $66(7), 681-697$. 
Figure 1. Operationalizing $\mathrm{RDoC}$ in a naturalistic treatment setting

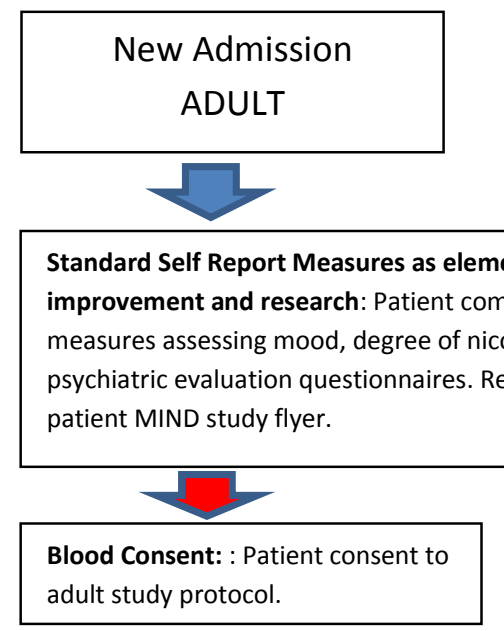

adult study protocol.

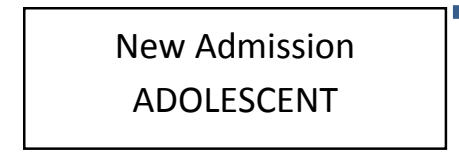

New Admission

ADOLESCENT

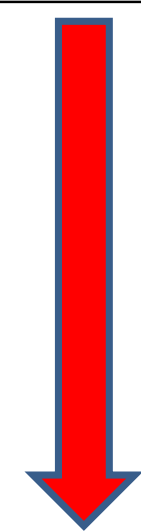

fMRI Consent: Patient consent to adult study protocol..
fMRI Eligibility Screen Form- fMRI personnel to verify eligibility for adult patients structural scan.

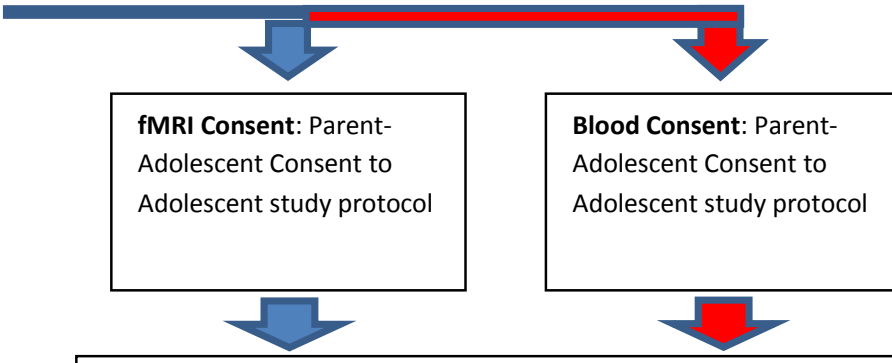

Standard Self Report Measures as elements of quality improvement and research: Patient completes base-line self-report measures assessing mood, degree of nicotine and other drug use psychiatric evaluation questionnaires. Research Assistant gives patient MIND study flyer.

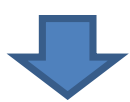

Adolescent Screening Form-fMRI personnel to verify eligibility for both adolescent patients

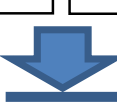

MRI Scan: Duration of the scan is approximately 40-50 minutes. Each subject will undergo the scanning session. Patients will be given a CD with .gif images of their brain taken during the

Blood Draw: Blood will be cryopreserved and stored at a secure location. If we are unable to collect a blood sample, a saliva sample may be used.
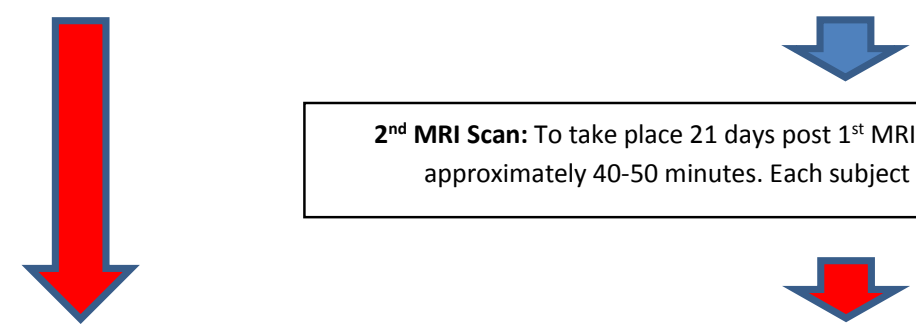

$2^{\text {nd }}$ MRI Scan: To take place 21 days post $1^{\text {st }}$ MRI procedure. The duration of the scan is approximately 40-50 minutes. Each subject will undergo the scanning session.

$\mathbf{2}^{\text {nd }}$ Blood Draw: To take place 21 days post $1^{\text {st }}$ blood draw. Blood will be cryopreserved and stored at a secure location. If we are unable to collect a blood sample, a saliva sample may be used. 